University of Nebraska - Lincoln

DigitalCommons@University of Nebraska - Lincoln

February 2007

\title{
The effect of interlayer interactions on the ferroelectric-paraelectric phase transition in multilayered thin films of vinylidene fluoride- trifluoroethylene copolymers
}

Jihee Kim

University of Nebraska - Lincoln

Hoydoo You

Argonne National Laboratory, Argonne, IL

Stephen Ducharme

University of Nebraska, sducharme1@unl.edu

Shireen Adenwalla

University of Nebraska-Lincoln, sadenwalla1@unl.edu

Follow this and additional works at: https://digitalcommons.unl.edu/physicsducharme

Part of the Physics Commons

Kim, Jihee; You, Hoydoo; Ducharme, Stephen; and Adenwalla, Shireen, "The effect of interlayer interactions on the ferroelectric-paraelectric phase transition in multilayered thin films of vinylidene fluoridetrifluoroethylene copolymers" (2007). Stephen Ducharme Publications. 31.

https://digitalcommons.unl.edu/physicsducharme/31

This Article is brought to you for free and open access by the Research Papers in Physics and Astronomy at DigitalCommons@University of Nebraska - Lincoln. It has been accepted for inclusion in Stephen Ducharme Publications by an authorized administrator of DigitalCommons@University of Nebraska - Lincoln. 
Published in Journal Of Physics: Condensed Matter 19 (2007) 086206 (21 pp); doi:10.1088/09538984/19/8/086206 Online at http://stacks.iop.org/JPhysCM/19/086206 Copyright $@ 2007$ IOP Publishing Ltd. Used by permission.

\title{
The effect of interlayer interactions on the ferroelectric-paraelectric phase transition in multilayered thin films of vinylidene fluoride- trifluoroethylene copolymers
}

\author{
Jihee Kim ${ }^{1}$, Hoydoo You $^{2}$, Stephen Ducharme ${ }^{1}$, and S \\ Adenwalla ${ }^{1}$ \\ ${ }^{1}$ Department of Physics and Astronomy and Nebraska Center for Materials and \\ Nanoscience, University of Nebraska, Lincoln, NE 68588-0111, USA \\ ${ }^{2}$ Material Science Division, Argonne National Laboratory, Argonne, IL 60439, \\ USA \\ Correspondence email: sducharme1@unl.edu
}

\begin{abstract}
The interaction between ferroelectric polymer films with different transition temperatures is evident in the effect of layer thickness on the ferroelectric- paraelectric phase transition in multilayer films, as revealed by $\mathrm{x}$-ray diffraction and dielectric measurements. The multilayer samples consisted of alternating Langmuir-Blodgett (LB) films of two different copolymers of vinylidene fluoride with trifluoroethylene, one with $80 \%$ vinylidene fluoride and a ferroelectric-paraelectric transition temperature on heating of $133 \pm 4{ }^{\circ} \mathrm{C}$ and the other with $50 \%$ vinylidene fluoride and a transition temperature of $70 \pm 4{ }^{\circ} \mathrm{C}$. Samples with a repeat period of 20 LB layers (10 contiguous layers of each composition) exhibited two distinct phase transitions, indicative of minimal interaction between the two materials. Films with a repeat period of 2, or films made from an equal mixture of the two copolymers, exhibited composite behavior, with an intermediate transition temperature and suppression of the transitions associated with the individual compositions. Films with a repeat period of 10 exhibit cross-over behavior. These results imply that the ferroelectric interaction length along the (110) direction, which is perpendicular to the film plane, is approximately $11 \mathrm{~nm}$.
\end{abstract}

\section{Introduction}

Studies of thin-film magnetic heterostructures have revealed a number of unexpected phenomena, some leading to useful technology. One notable example is the giant magnetoresistive (GMR) effect stemming from the oscillatory interaction of magnetic layers separated by non-magnetic spacers a few nanometers thick [1]. GMR read heads were quickly developed and were instrumental in increasing magnetic disk drive capacities. Studies of 
superlattices of antiferromagnetic $\mathrm{CoO}$ and $\mathrm{NiO}$ show evidence of the influence of spin polarization at the interface - the ordering temperature of the superlattice is intermediate between the Néel temperatures of the constituent layers [2,3]. Thin antiferromagnetic films sandwiched between ferromagnetic layers show a large increase in the Néel temperature, due to polarization effects from the ferromagnetically ordered spacers [4]. These interaction effects occur over much longer length scales than the exchange coupling that produces the ordered ferromagnetic and antiferromagnetic states.

The role of finite-size effects in ferroelectric materials is less clear, though of critical importance in the rapidly growing ferroelectric memory industry, where the bistability of polarization of thin ferroelectric films is paramount. Analyses using mean-field theory generally conclude that the depolarization field energies and surface energies will suppress ferroelectricity $[5,6]$. There were several early observations of finite-size effects in ferroelectric oxide thin films (as thin as $100 \mathrm{~nm}$ ) [7-9], and of nanoparticles as small as $25 \mathrm{~nm}[10,11]$, although these results were probably dominated by variations in sample composition or microstructure [12]. Improvements in the fabrication of thin perovskite films pushed the limit below $15 \mathrm{~nm}$ [13-17], in accordance with the predictions of firstprinciples calculations $[18,19]$. Ferroelectricity has been reported in films as thin as 1 $\mathrm{nm}$ in both polymers [20] and oxides [21]. The most remarkable results of the thin-film studies were that reduced thickness does not necessarily decrease the Curie temperature or spontaneous polarization. Though polarization measurements of the thinnest ferroelectric polymers are inconclusive [20], a recent study of $\mathrm{BaTiO}_{3}$ thin films with $\mathrm{SrRuO}_{3}$ electrodes showed significant polarization decline only below $15 \mathrm{~nm}$ [22], and studies of unit cell tetragonality in $\mathrm{PbTiO}_{3}$ films [23] also indicated a significant decrease in polarization below $10 \mathrm{~nm}$ in thickness.

Studies of ferroelectric multilayers and superlattices, composed of ferroelectric oxides with different bulk transition temperatures $\left(T_{\mathrm{C}} \mathrm{s}\right)$ or of ferroelectric and paraelectric materials, shed additional light on the thin-film stability puzzle and on the interaction length of polarization correlation. There have been numerous experimental studies of superlattices involving ferroelectric materials, mostly in the past five years. Theoretical modeling has been effective in guiding research in ferroelectric superlattices, predicting interlayer polarization interactions [24], $T_{\mathrm{C}}$ enhancement [25], and enhanced dielectric response $[5,6,26]$. Experimental results do reveal $T_{\mathrm{C}}$ enhancement with a critical thickness of order $10 \mathrm{~nm}$ or less in ferroelectric superlattices of $\mathrm{KTaO}_{3} / \mathrm{KNbO}_{3}$ [27], in $\mathrm{BaTiO}_{3} /$ $\mathrm{SrTiO}_{3}[28,29]$, and $\mathrm{PbTiO}_{3} / \mathrm{SrTiO}_{3}$ [30]. In addition, superlattices of two different paraelectric materials show evidence of both antiferroelectric and ferroelectric ordering [31], while superlattices consisting of ferroelectric and antiferroelectric layers show interesting intermediate behavior [32].

Strain can play a major role in epitaxial superlattices when the transition is substantially displacive, as for the oxide studies just mentioned, and other studies of epitaxial films [33-35]. There is also evidence for correlations between the critical superlattice period and the domain size [27]. The influence of strain is likely much smaller in the much softer ferroelectric polymers. For example, an all-organic composite of copper-phthalocyanine (a high dielectric constant material) with ferroelectric $\mathrm{P}(\mathrm{VDF}-\mathrm{TrFE})$, copolymers of vinylidene fluoride (VDF) with trifluoroethylene (TrFE), which shows greatly enhanced dielectric properties [36], which are better explained by short-range exchange interactions at the interface between the two materials, and not by long-range strain effects [37]. The size and length scales are not precisely defined in the composites, and hence interaction information is averaged over a wide range of length scales. Precision deposition of ferroelectric and dielectric multilayers can better control interactions and possibly produce significant enhancement of key functional properties critical to applications such as non-volatile memory, infrared imaging, and electromechanical transducers. 
The ability to tune the ferroelectric properties of nanostructured materials provides some appealing technological opportunities. Ferroelectric superlattices have exhibited enhanced dielectric constants attributed to polarization correlations [26, 38, 39] or to purely dielectric effects [40], and enhanced nonlinear optical response [22, 28, 39], demonstrating the potential technological benefits of ferroelectric crystal engineering. Other nanoscale structures studied to date, including arrays of pillars [41] and nanotubes [42-45], demonstrate additional means of controlling bulk properties through nanostructure.

Our motivation for this work was to investigate the interaction between ferroelectric polymer layers with different $T_{\mathrm{C}} \mathrm{S}$ in a well-defined structure that is relatively free of strain. Varying the composition and layer thickness should probe several key features of ferroelectric interactions, namely, whether or not ferroelectric layers couple across a paraelectric layer, whether it is possible to induce ferroelectricity in a paraelectric layer without first inducing strain, and what length scales are important for this interaction to occur. In order to accomplish this we chose a multilayered structure consisting of alternating layers of two $\mathrm{P}(\mathrm{VDF}-\mathrm{TrFE})$ copolymers with different $T_{\mathrm{C}} \mathrm{s}$. The $\mathrm{P}(\mathrm{VDF}-\mathrm{TrFE})$ copolymers have nearly the same spontaneous polarization, of order $0.1 \mathrm{C} \mathrm{m}^{-2}$, and a composition-dependent $T_{\mathrm{C}}$ ranging from 65 to $208{ }^{\circ} \mathrm{C}$, for compositions containing $40 \%$ VDF to $100 \%$ VDF, respectively [46]. The compositions P(VDF-TrFE 50:50) and P(VDF-TrFE 80:20) were chosen for the present study as the two constituents of the multilayer, since they have well-separated bulk $T_{\mathrm{C}} \mathrm{s}$ (measured on heating) of approximately $65{ }^{\circ} \mathrm{C}$ and $142{ }^{\circ} \mathrm{C}$ [46], respectively, and therefore are good candidates for exploring interlayer interaction effects. We chose these values because compositions with more than $80 \%$ VDF do not crystallize well and have $T_{\mathrm{C}} \mathrm{s}$ inconveniently located above their melting points. Copolymers with less than $50 \%$ VDF have so far proven too variable in their ferroelectric properties to provide reliable samples.

Here we report a study of the structure and ferroelectric properties of multilayers of two ferroelectric copolymers, P(VDF-TrFE 80:20) and P(VDF-TrFE 50:50), with repeat periods of 2, 10 and 20 LB layers, and a constant total thickness of 20 LB layers. The results from these samples were also compared with pure films composed only of a single copolymer and films made from a 1:1 mixture of the two compositions. The two-dimensional nature of the ferroelectric transition [20,47] presumably rules out direct ferroelectric interaction between the various layers; however, interactions via the polarization of the intervening layer may still occur. For each sample, we measured the lattice spacing and the dielectric constant as a function of temperature on both heating and cooling. The films with the repeat period 2, which consisted of one LB layer of each composition, exhibit intermediate behavior similar to that of the mixture. Films with repeat period 20, which consisted of contiguous 10 LB layers of each composition, behave like two independent films. The cross-over appears to be with the sample with repeat period 10, which alternates five layers of the 50:50 copolymer with five layers of the 80:20 copolymer.

\section{Sample preparation}

The ultrathin ferroelectric polymer films were deposited by Langmuir-Blodgett (LB) deposition using procedures described in detail elsewhere [47-50] LB deposition affords ultrathin films with good crystallinity [51] and stable ferroelectric properties, such as spontaneous polarization and Curie temperature, that are only weakly dependent on thickness $[20,47]$. Briefly, deposition proceeded as follows. We spread a solution of the polymer $(0.05 \%$ weight concentration in dimethyl sulfoxide) onto the surface of an ultrapure water (18 $\mathrm{M} \Omega \mathrm{cm}$ resistivity) subphase maintained at $25^{\circ} \mathrm{C}$. The polymer surface film was compressed slowly to a surface pressure of $5 \mathrm{mN} \mathrm{m}^{-1}$, and then transferred to the substrate by horizontal dipping at a slight angle to control the line of contact. The film thickness 
(a)

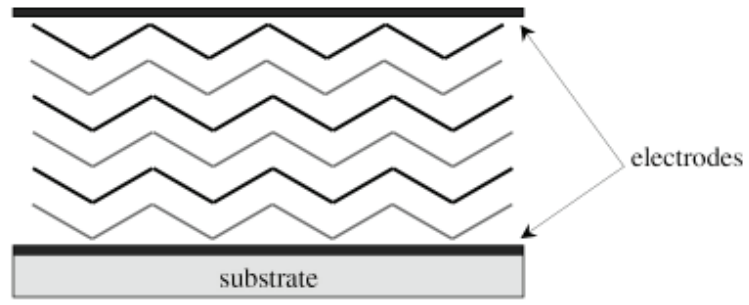

(b)

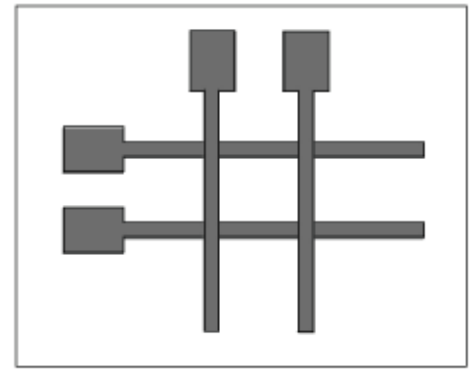

Figure 1. Schematic diagram of sample structure. (a) Layering of the LB films shown between the top and the bottom electrode. The darker lines indicate layers of 50:50 copolymer, the lighter lines indicate layers of 80:20 copolymer. (b) Layout of a capacitor sample

was controlled by repeating the LB layer transfer, and multilayered samples were made by alternating between two isolated trough sections containing the two polymer compositions, each held at the same temperature and surface pressure. Completed samples were annealed for $2 \mathrm{~h}$ at $135^{\circ} \mathrm{C}$ to optimize their crystal structure and stabilize the ferroelectric properties [47, 52].

The samples for the present work consisted of homogeneous and multilayer LB films of two $\mathrm{P}(\mathrm{VDF}-\mathrm{TrFE})$ copolymers, one with $80 \% \mathrm{VDF}$, designated 80:20, and the other with 50\% VDF, designated 50:50. The LB films were deposited on two different substrates, depending on the intended measurement. Samples for x-ray diffraction (XRD) measurements consisted of LB films deposited directly on (100) silicon wafers. Capacitors for dielectric measurements were fabricated by evaporating bottom aluminum strip electrodes on a glass microscope slide, then depositing the LB film or multilayer, and evaporating aluminum strip top electrodes oriented orthogonal to the bottom electrodes. The completed capacitor, shown schematically in figure 1, consists of four individually addressable spots.

Measurements were made on six pairs of samples, each pair consisting of one sample for XRD measurements and another for dielectric measurements, made as described above. The LB films on all the samples consisted of 20 LB layers (20 individual transfers) but differ in the composition and sequence of the LB layers. One pair of homogeneous reference samples was made with each of the two copolymers. Three pairs of multilayer samples consisted of equal-thickness alternating layers of the two copolymers with repeat periods of 2, 10, and 20 LB layers. An additional pair of samples was made from a solution containing equal amounts by weight of the two copolymers. The complete list of samples and their compositions is given in table 1.

\section{Experimental methods}

The breadth of the ferroelectric-paraelectric phase transition and inherent thermal hysteresis due to phase coexistence make it difficult to determine the true phase transition tem- 
Table 1. Sample composition and basic properties measured as described in the text. The sample thicknesses were obtained from the x-ray reflectivity data shown in figure 3 . The thermal expansion coefficients were obtained from the slopes of the lines in figure 7 for both ferroelectric and paraelectric phases.

\begin{tabular}{|c|c|c|c|c|c|}
\hline \multirow[b]{2}{*}{ Sample } & \multirow[b]{2}{*}{ Composition } & \multirow{2}{*}{$\begin{array}{l}\text { Repeat } \\
\text { period } \\
\text { (LB layers) }\end{array}$} & \multirow{2}{*}{$\begin{array}{l}\text { Sample } \\
\text { thickness } \\
(\mathrm{nm})^{\mathrm{a}}\end{array}$} & \multicolumn{2}{|c|}{$\begin{array}{l}\text { Thermal expansion coefficient } \\
\qquad(\mathrm{d} L / \mathrm{d} T) / L_{0}\left({ }^{\circ} \mathrm{C}\right)^{-1}\end{array}$} \\
\hline & & & & $\begin{array}{l}\text { Ferroelectric } \\
\text { phase }\end{array}$ & $\begin{array}{l}\text { Paraelectric } \\
\text { phase }\end{array}$ \\
\hline $50: 50$ & $\begin{array}{l}50: 50 \\
\text { copolymer }\end{array}$ & - & 50 & $3.7 \times 10^{-3}$ & $1.3 \times 10^{-3}$ \\
\hline $80: 20$ & $\begin{array}{l}80: 20 \\
\text { copolymer }\end{array}$ & - & 51 & $1.3 \times 10^{-3}$ & $1.1 \times 10^{-3}$ \\
\hline Period 20 & $\begin{array}{l}\text { Alternating } \\
10 \text { LB layers }\end{array}$ & 20 & 48 & $\begin{array}{l}2.8 \times 10^{-3 b} \\
0.8 \times 10^{-3 b}\end{array}$ & $1.4 \times 10^{-3}$ \\
\hline Period 10 & $\begin{array}{l}\text { Alternating } \\
5 \text { LB layers }\end{array}$ & 10 & 42 & $1.6 \times 10^{-3}$ & $1.3 \times 10^{-3}$ \\
\hline Period 2 & $\begin{array}{l}\text { Alternating } \\
1 \text { LB layers }\end{array}$ & 2 & 46 & $0.7 \times 10^{-3}$ & $1.0 \times 10^{-3}$ \\
\hline Mixture & $\begin{array}{l}\text { Equal weights } \\
50: 50 \& 80: 20\end{array}$ & - & 51 & $1.2 \times 10^{-3}$ & $1.1 \times 10^{-3}$ \\
\hline
\end{tabular}

\footnotetext{
${ }^{a}$ The sample thickness was determined from a linear least-squares fit of the positions of the minima in figure 3 , with a root-mean-square statistical uncertainty of $5 \%$ or less for all fits.

${ }^{\mathrm{b}}$ The two thermal expansion coefficients for the period 20 sample are due to the separate behavior of 80:20 and 50:50 layers in the sample; the values $2.8 \times 10^{-3}$ and $0.8 \times 10^{-3}$ are from 50:50 layers and 80:20 layers, respectively.
}

perature. Therefore, we use both dielectric measurements and x-ray diffraction to investigate the behavior. The advantage of $\mathrm{x}$-ray diffraction is that it provides a quantitative measure of the amount of material in the ferroelectric and paraelectric states in the phase coexistence region $[51,53,54]$. As we shall see, this enables us to clearly define an effective phase transition temperature and also chart the evolution of phase conversion with temperature and history. Since the x-ray scattering lengths of the two compositions are almost identical, we were not able to directly probe the multilayer period. However, as we shall see subsequently, the behavior of the differently structured samples is quite distinct, implying that the structure of the superlattice is preserved to a large extent.

Sample crystallinity was measured by x-ray diffraction (XRD) in the $\theta-2 \theta$ geometry, which measures the crystal spacing normal to the substrate. Previous studies have shown that the LB films are oriented with the (110) planes parallel to the substrate, meaning that the chains, which are along the [001] direction, are in the film plane and the spontaneous polarization in the ferroelectric phase, which is along the [010] direction is tilted $30^{\circ}$ away from the normal $[51,54]$. The XRD peaks near $18^{\circ}$ and $19^{\circ}$ at an X-ray wavelength $\lambda=$ $1.54 \AA$ are the (110) diffraction peaks in the paraelectric and ferroelectric phases, respectively [55]. These peaks have the same structure factor, so the integrated intensities give the relative fraction of material in each phase [54].

Initial x-ray diffraction measurements were performed on a set of preliminary samples to determine the sample crystallinity and follow the progress of the phase transition as they were heated. These measurements were made on a Rigaku $\theta-2 \theta$ diffractometer with a fixed $\mathrm{Cu} \mathrm{K} \alpha$ cathode. This initial XRD measurement showed relatively low crystallinity of the samples before annealing and considerable improvement after annealing, as shown in figure 2 . For the 50:50 and 80:20 samples the crystallinity improved by a factor 


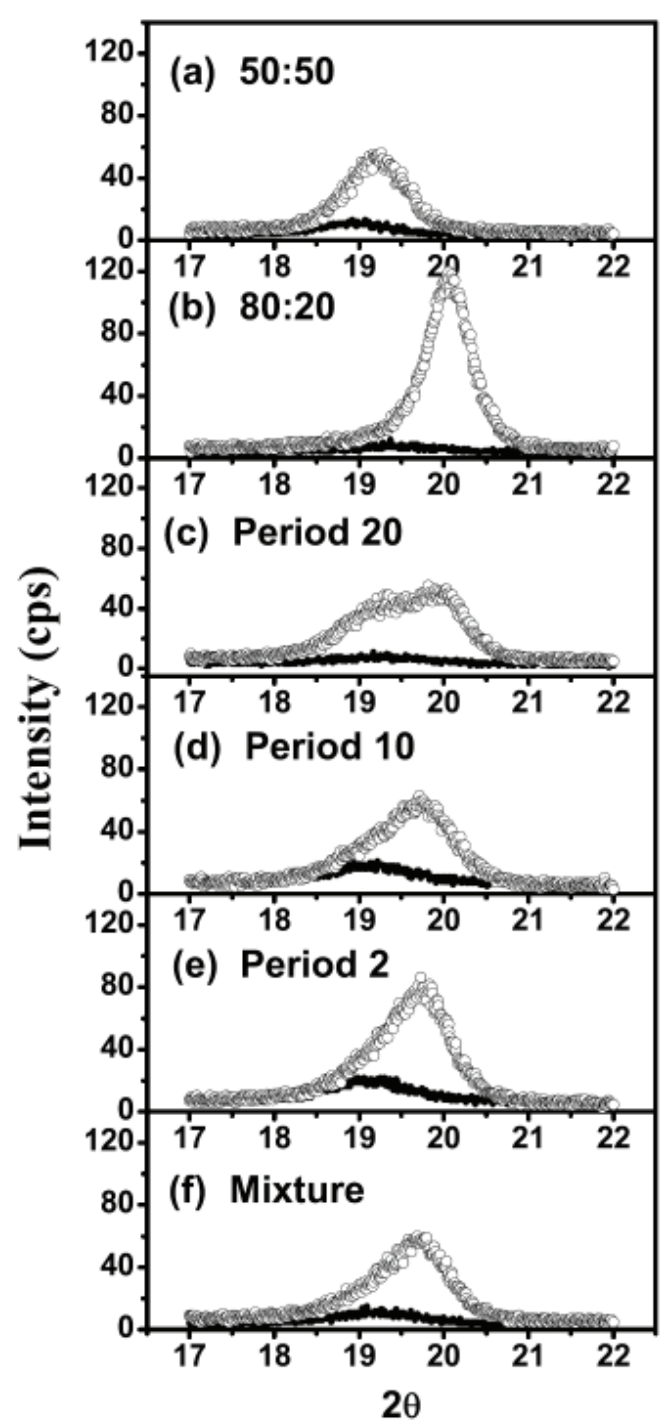

Figure 2. X-ray diffraction data from the XRD samples recorded at room temperature with the Rigaku $\theta-2 \theta$ instrument both before (solid circles) and after (open circles) annealing.

of 5 and 12 respectively as measured by the area under the diffraction peaks. The samples were annealed by heating them from room temperature to $135^{\circ} \mathrm{C}$ at a rate of $1{ }^{\circ} \mathrm{C} \mathrm{min}{ }^{-1}$, held at $135^{\circ} \mathrm{C}$ for $2 \mathrm{~h}$ and cooled down to room temperature at the same rate. Both prior studies $[51,52]$ and present results indicate that this annealing procedure optimizes the degree of crystallinity. Each sample was then enclosed in a temperature-controlled copper chamber, which was mounted on a goniometer. The chamber operated at atmospheric pressure. The sample temperature was accurate within $2{ }^{\circ} \mathrm{C}$. The sample temperature was increased at the rate of $1{ }^{\circ} \mathrm{C} \mathrm{min}{ }^{-1}$. Measurements were made at intervals of $20{ }^{\circ} \mathrm{C}$ after holding for $20 \mathrm{~min}$ at each set temperature. An identical set of samples was then made for the detailed XRD studies performed at the synchrotron.

The total thickness of each film was measured after completion of the synchrotron studies by grazing-incidence $\theta-2 \theta$ x-ray reflectivity on a Bruker AXS diffractometer. 


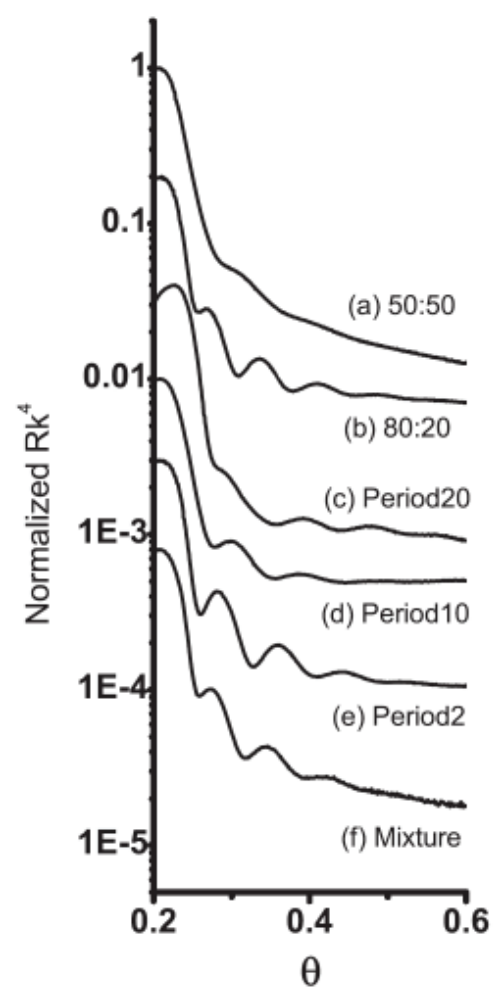

Figure 3. X-ray reflectivity data recorded with the Bruker $\theta-2 \theta$ instrument showing thickness oscillations for the indicated samples. The curves have been offset vertically for clarity. All except for the 50:50 sample were the same as those used for the synchrotron x-ray diffraction studies. The original 50:50 sample was unavailable for the x-ray reflectivity studies, so the sample prepared for the primary check-up study before XRD measurement at the synchrotron was used. Due to small number of fringes with this particular sample, an additional sample was also prepared under same conditions, and the thicknesses from both samples are the same within experimental uncertainty.

These data were analyzed as follows. The x-ray reflectivity $R$ was multiplied by $k^{4}$, where

$$
k=\frac{2 \pi}{\lambda} \sin \theta
$$

is the scattering vector, in order to account for the effects of the Fresnel reflectivity. The modified reflectivity data for all six samples are shown in figure 3. The presence of multiple oscillations indicates relatively smooth interfaces. The sample thickness was determined by using the modified Bragg equation,

$$
\left(m+\frac{1}{2}\right) \lambda=2 t \sqrt{\sin ^{2} \theta_{m}-\theta_{\mathrm{c}}^{2}},
$$

for the positions of the diffraction minima, where $m$ is an integer index for the diffraction order, $\lambda$ is the x-ray wavelength ( $1.54 \AA$ in this case), $t$ is the film thickness, $\theta_{m}$ is the angle corresponding to the diffraction minimum of index $m$ [56], and $\theta_{\mathrm{c}}=0.2^{\circ}$ is the critical angle of the copolymer for $\mathrm{x}$-rays with wavelength $1.54 \AA$. The small differences in scattering length densities between the two copolymers implies that the multilayered samples may be treated as a single uniform layer for the purposes of this thickness measurement. The thicknesses determined by $\mathrm{x}$-ray reflectivity are listed in table 1 . The sample thicknesses for the pure 80:20 and 50:50 samples and the mixture compositions are approximately $50 \mathrm{~nm}$, while the multilayer samples are slightly thinner, with an average thickness 
of $45 \mathrm{~nm}$, slightly thinner than the pure compositions. According to these thickness measurements, each transfer of LB film from the water subphase consists of approximately six molecular layers per LB transfer. The roughness is estimated to be approximately $2 \mathrm{~nm}$, as seen from previous AFM measurements on $\mathrm{P}(\mathrm{VDF}-\mathrm{TrFE}$ 70:30) copolymer films fabricated under the same conditions [57].

The bulk of the x-ray diffraction measurements were made at the Advanced Photon Source at Argonne National Laboratory, on the 12-BM/BESSRC-CAT beam line, which operated at a wavelength $\lambda=1.0 \AA$ with a $2 \theta$ angular resolution of $0.012^{\circ}$. The sample was placed in vacuum on the cold finger of a recirculating gas refrigerator that was mounted on the diffractometer goniometer. The sample temperature was accurate to within $1{ }^{\circ} \mathrm{C}$. The diffraction measurements were recorded on both heating and cooling over the range -5 to $+125{ }^{\circ} \mathrm{C}$, at temperature intervals of $5{ }^{\circ} \mathrm{C}$ far from the phase transition temperature, and intervals of $3{ }^{\circ} \mathrm{C}$ near it. Beginning the measurements well below room temperature ensured that the samples were initially in the ferroelectric phase.

The dielectric constant of a ferroelectric exhibits peaks at the transition between the paraelectric and ferroelectric phases. These peaks are due to the large polarizability near the phase transition. Capacitance measurements were made with a Hewlett-Packard 4192A impedance analyzer operating at a frequency of $1 \mathrm{kHz}$ and an amplitude of $0.1 \mathrm{~V}$. The samples were mounted in a temperature-controlled copper chamber at atmospheric pressure. The sample temperature was accurate within $1{ }^{\circ} \mathrm{C}$. The sample capacitance was continuously recorded as the sample was heated at a rate of $1{ }^{\circ} \mathrm{C} \mathrm{min}-1$ from room temperature to $125^{\circ} \mathrm{C}$ and while cooling back to room temperature at the same rate. The exception was the pure $80: 20$ composition sample which was heated to $135^{\circ} \mathrm{C}$.

The switching behavior of the capacitor samples was characterized at room temperature by measuring the capacitance as a bias voltage was slowly varied between opposing polarization saturation levels. The capacitance hysteresis data shown in figure 4 demonstrates that all the samples exhibit the characteristic "butterfly" shape, with capacitance peaks indicating polarization switching. The butterfly curves repeat on continued cycling and are typical of those obtained with the ferroelectric copolymer LB films [47, 58]. The magnitude of the coercive voltage varies slightly among the samples, but does not seem to be correlated with the layered structure. This is to be expected, because the coercive field of the copolymers depends only weakly on composition [59].

\section{Results and discussion}

\subsection{Structure}

The ferroelectric-to-paraelectric conversion is accompanied by a change in the crystal structure from the all-trans conformation of the ferroelectric phase to the trans-gauche paraelectric phase. Figure 5 shows the $\theta-2 \theta$ XRD data from the synchrotron recorded at the lowest $\left(-5^{\circ} \mathrm{C}\right)$ and highest $\left(+125^{\circ} \mathrm{C}\right)$ temperatures for all the samples. The peaks near $2 \theta$ $=13^{\circ}$ correspond to a (110) $d$-spacing of $4.4 \AA$ for the ferroelectric phase, while the peaks near $2 \theta=11.9^{\circ}$ correspond to a $d$-spacing of $4.8 \AA$ for the paraelectric phase. The lattice expansion of $10 \%$ from the ferroelectric to paraelectric phases is due to the increased size of the chains with trans-gauche conformation [55]. The pure 80:20 sample and the period 20 sample have noticeable amounts of residual ferroelectric phase at $125^{\circ} \mathrm{C}$, due to phase coexistence and the relatively high transition temperature of the 80:20 copolymer, a point that will be discussed further below.

The lattice spacing in the paraelectric phase is only weakly dependent on composition, as the peak positions of the pure 50:50 and pure 80:20 samples at $125{ }^{\circ} \mathrm{C}$ were nearly identical, at $11.86 \pm 0.02^{\circ}(4.835 \pm 0.005 \AA)$ and $11.95 \pm 0.02^{\circ}(4.80 \pm 0.01 \AA)$ re- 

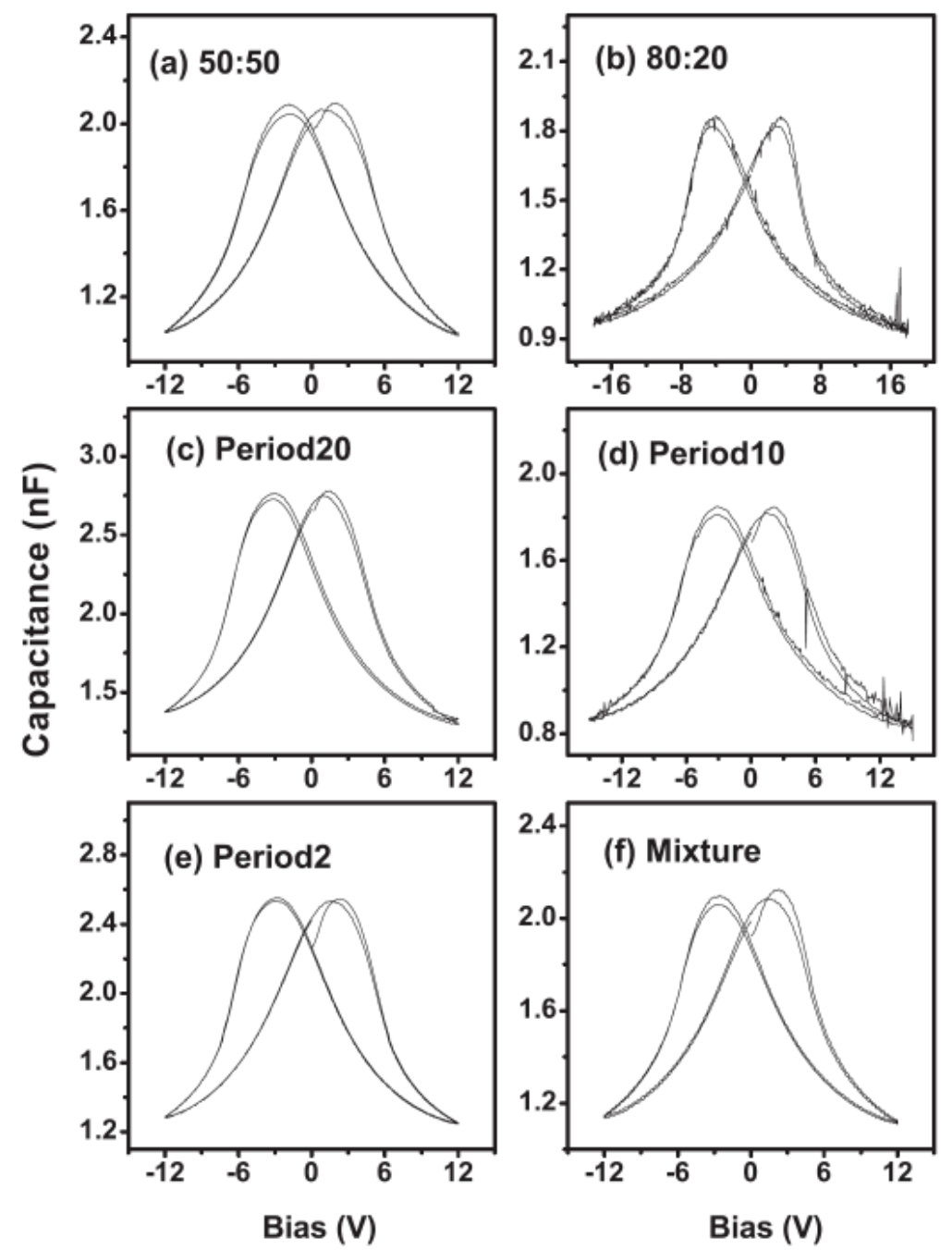

Figure 4. The "butterfly" hysteresis curves of the capacitor samples measured at a frequency of $1 \mathrm{kHz}$ and amplitude $0.1 \mathrm{~V}$ while cycling the bias voltage at a rate of $6 \mathrm{~V}$ $\min ^{-1}$.

spectively, effectively irresolvable because of the peak width of approximately $0.4^{\circ}$. The tightly packed ferroelectric phase, on the other hand, is more sensitive to the presence of the extra fluorine atoms on the hydrogen side of the backbone. At $-5{ }^{\circ} \mathrm{C}$, the peak positions of the pure 50:50 and pure 80:20 samples are readily resolved, at $12.86 \pm 0.02^{\circ}(4.46$ $\pm 0.01 \AA)$ and $13.48 \pm 0.02^{\circ}(4.26 \pm 0.005 \AA)$, respectively.

\subsection{X-ray diffraction results}

Figure 6 shows the temperature evolution of the (110) peaks for the six XRD samples from -5 to $125^{\circ} \mathrm{C}$ on heating. The transition between the ferroelectric and paraelectric phases in the VDF copolymers is gradual, owing to the first-order nature of the transition [60]. The breadth of the phase transition is not sensitive to sample defects or inhomogeneity, as is evident by comparing studies of polymorphous lamellar samples [61] with highquality linear-chain crystals of VDF copolymers [62]. Moreover, the phase transition is 

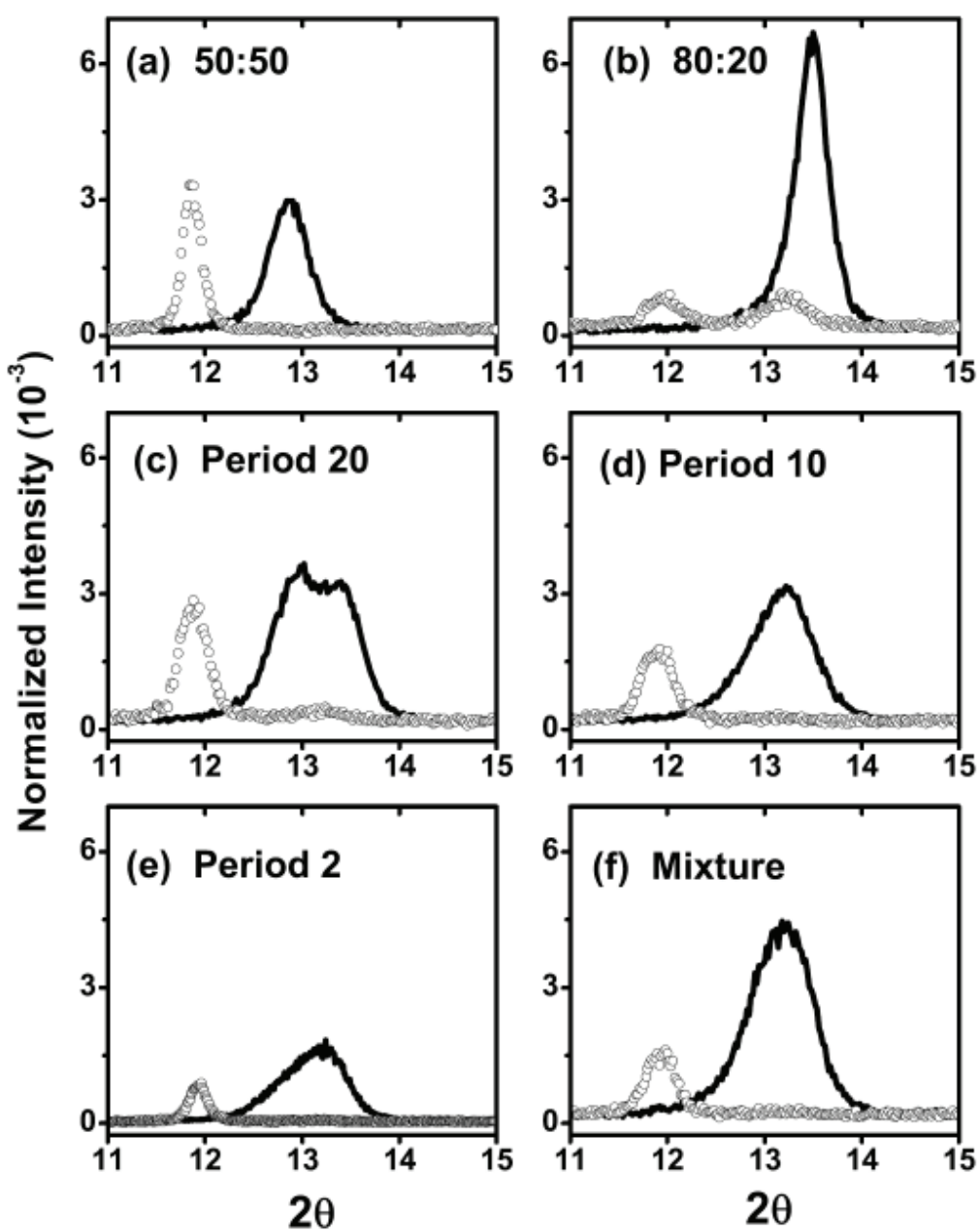

Figure 5. Comparison between $\mathrm{x}$-ray diffraction data recorded at the synchrotron at -5 ${ }^{\circ} \mathrm{C}$ (solid lines) and at $125^{\circ} \mathrm{C}$ (open circles) for all the samples. The peaks near $12^{\circ}$ are the (110) peaks from the paraelectric phase, while the peaks between $13^{\circ}$ and $14^{\circ}$ are the (110) peaks from the ferroelectric phase.

highly hysteretic, so the transition temperatures on heating and cooling are different. We will focus on the phase changes observed during heating after stabilization at room temperature or below, because this produces the best repeatability in the initial phase proportions. The proportion of each phase is readily quantified by the intensity of the (110) diffraction peaks, the ferroelectric peak at approximately $13^{\circ}$ and the paraelectric peak at approximately $12^{\circ}$. All samples exhibit a large coexistence region, the range of temperatures where both phases persist. The pure 50:50 sample (see figure 6(a)), having the lower transition temperature, shows essentially full conversion to the paraelectric phase by approximately $80^{\circ} \mathrm{C}$, while the pure $80: 20$ sample (see figure $6(\mathrm{~b})$ ) is not yet fully converted at $125{ }^{\circ} \mathrm{C}$. These two pure samples provide a useful reference for comparison. For example, the XRD data from the period 20 sample resembles a superposition of the structures of the two component materials, with two distinct ferroelectric phase XRD peaks recorded at $-5{ }^{\circ} \mathrm{C}$ and a two-stage transition to the paraelectric phase. The mixture sample (see figure 6(f)), the period 2 sample (figure 6(c)), and the period 10 sample (figure 6(d)) 

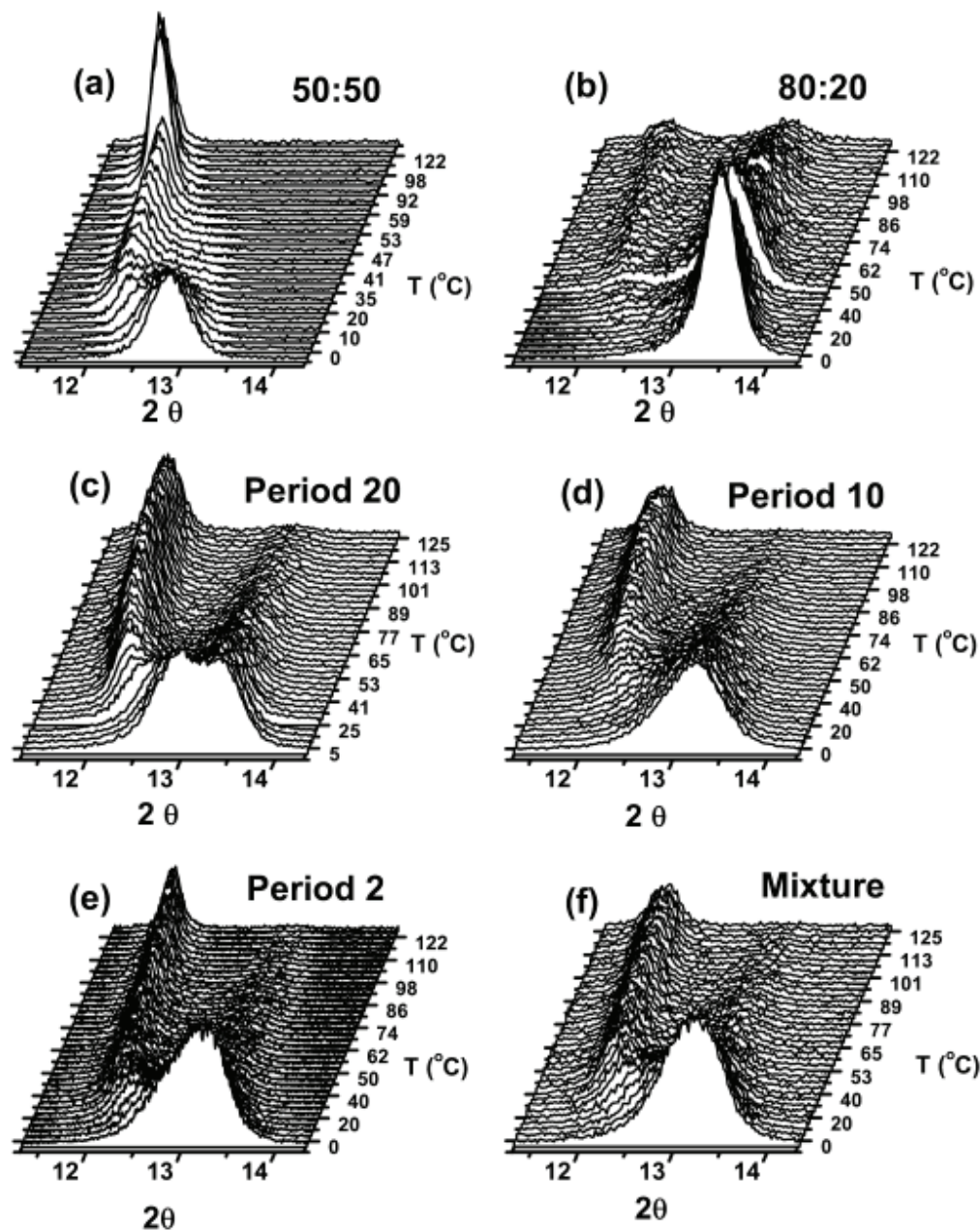

Figure 6. The synchrotron $\mathrm{x}$-ray diffraction data as function of temperature, showing the ferroelectric-to-paraelectric phase conversion for the following 20 LB layer films: (a) pure 50:50 copolymer; (b) pure 80:20 copolymer; (c) period 20; (d) period 10; (e) period 2; and (f) mixture.

show nearly full conversion to the paraelectric phase by $125^{\circ} \mathrm{C}$. On the other hand, both the pure 80:20 sample and the period 20 sample retain some of the paraelectric phase, even at $125^{\circ} \mathrm{C}$, as is evident in figures $5(\mathrm{~b})$ and (c), indicating that the 80:20 layers in the period 20 sample are relatively unperturbed.

To make a more quantitative comparison, we fitted each XRD measurement to a set of peaks with a Gaussian line shape (except for the period 20 sample, where a Lorentzian line shape resulted in higher quality fits). With such thin films and inherently broad diffraction peaks, the fitting results do not necessarily determine unambiguously which spacings are present. An elementary model calculation of the expected diffraction peak heights, widths and positions for the multilayer samples (assuming planar interfaces and perfect lattice coherence through the thickness of the sample) indicates that the (110) peaks for the ferroelectric structures of the 50:50 and 80:20 copolymers should produce a single irresolvable peak in the period 2 sample, whereas in the period 20 sample, the separate peaks should be clearly distinguishable. (The peak widths, which are of the order of $1^{\circ}$ in $2 \theta$, are much greater than the $0.012^{\circ}$ resolution of the APS diffractometer.) Note that 


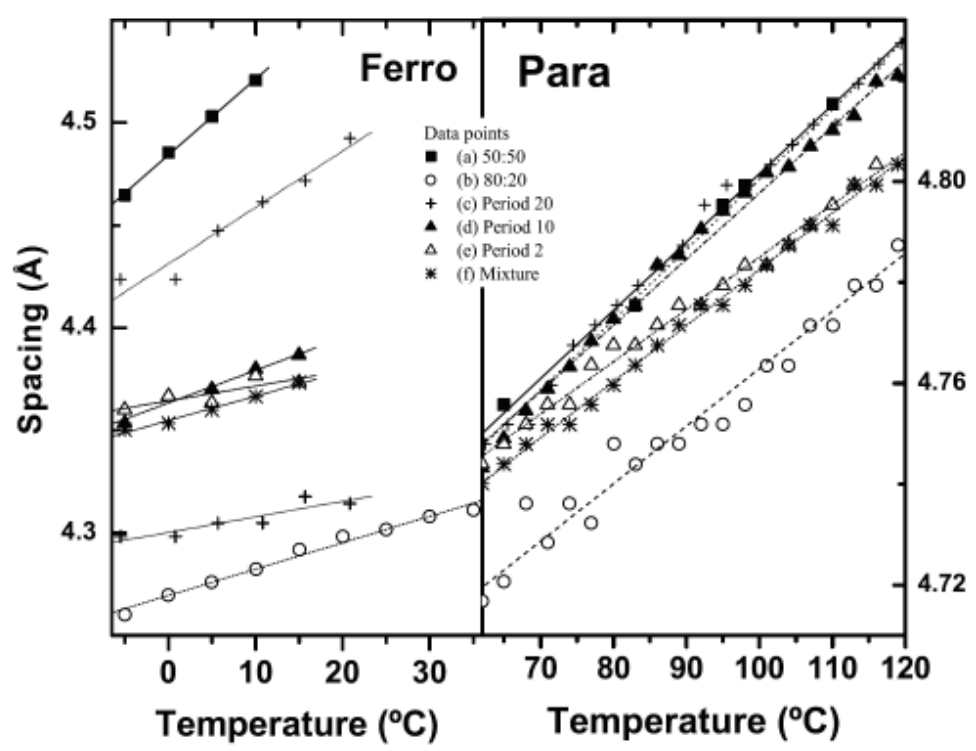

Figure 7. Lattice spacings determined from the x-ray diffraction peaks shown in figure 6 as a function of temperature, for the following samples: (a) pure 50:50 (solid squares); (b) pure 80:20 (open circles); (c) period 20 (crosses); (d) period 10 (solid triangles); (e) period 2 (open triangles); and (f) mixture (asterisks). The lines represent linear regression fits to the peak positions yielding the thermal expansion coefficients, which are listed in table 1 .

the model shows that an apparent merging of the (110) XRD peaks in films with small repeat periods is purely structural - it does not necessarily indicate that the period 2 sample, for example, behaves as a composite or mixed sample (though this indeed may be the case), only that the two peaks are structurally indistinguishable. In all but the period 20 sample, the two-peak fits (one peak from the ferroelectric phase and only one from the paraelectric phase) were much better, meaning that distinct ferroelectric peaks for two independent materials could not be resolved. For the period 20 sample, we obtained better fits with three peaks, one for each ferroelectric peak from the two compositions and a single combined paraelectric peak. (The paraelectric peaks are at nearly the same spacing, and so are not resolvable, as we confirmed by attempting to fit two paraelectric peaks.) The peak positions obtained from this analysis are shown in figure 7 . We calculated the integrated intensity of each peak as obtained from the fitting procedure, and normalized the total intensity to 1 at each temperature, so the values represent the fraction of the ferroelectric and paraelectric phases, respectively. The normalized intensities obtained in this fashion are shown in figure 8 .

The lattice spacings of all samples obtained from the peak positions of the x-ray diffraction data shown in figure 6 are plotted in figure 7 as a function of temperature. The thermal expansion coefficients for both the ferroelectric and paraelectric phases of all samples were obtained and are listed in table 1. Regardless of the values of the expansion coefficients, the lattice spacings of all the heterogeneous samples are intermediate between those of the pure 50:50 and pure 80:20 copolymers, for both phases. In the paraelectric phase, both the spacing and the thermal expansion coefficients of the samples are within a small range, $\sim 1 \%$ and $\sim 30 \%$, respectively. In the ferroelectric phase, the lattice spacings and the thermal expansion coefficients of the samples cover a wider range, $\sim 5 \%$ and $\sim 300 \%$, respectively. The thermal expansion of the ferroelectric and paraelectric peaks in the period 10 sample are intermediate between the corresponding values for the two pure 


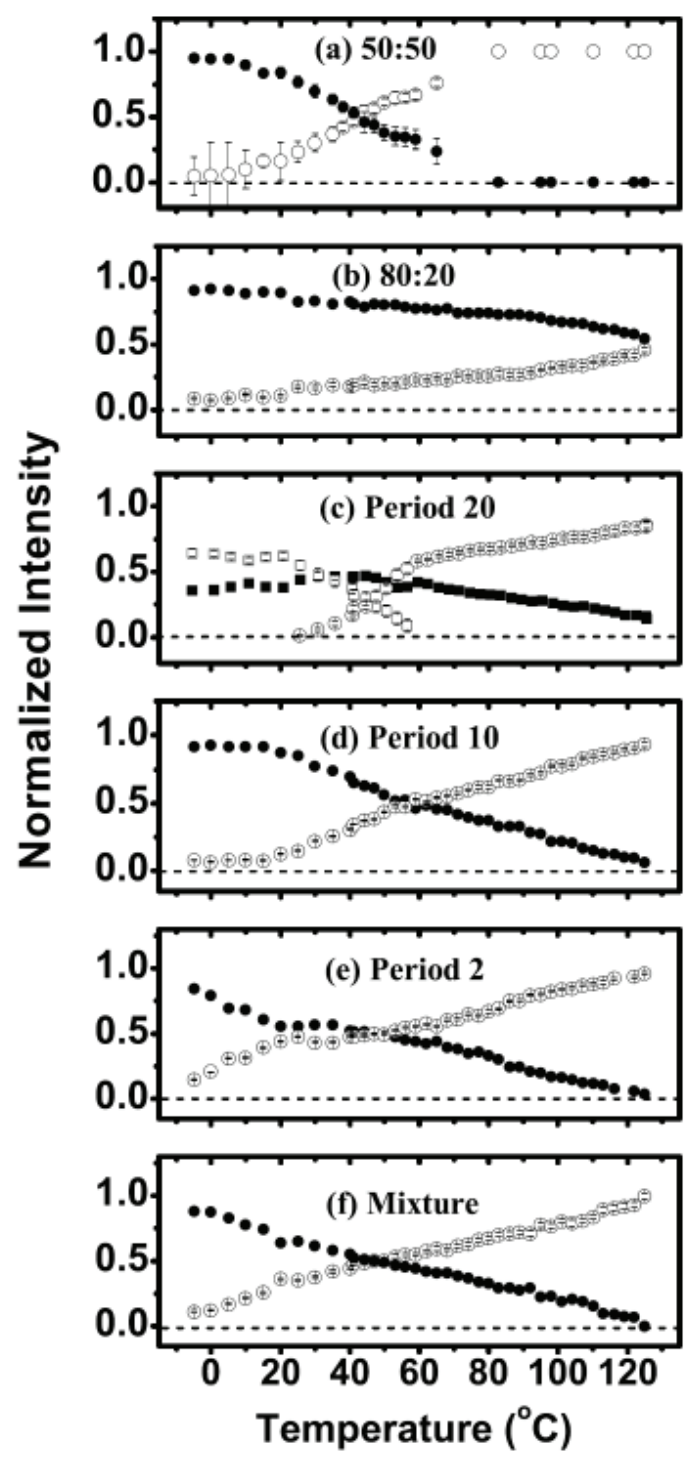

Figure 8. The normalized peak intensity as a function of temperature obtained from analysis of the $\mathrm{x}$-ray diffraction data shown in figure 6 . The graphs shown are for the six samples as follows: (a) pure 50:50; (b) pure 80:20; (c) period 20; (d) period 10; (e) period 2; and (f) mixture. Open circles indicate the paraelectric phase, and solid circles indicate the ferroelectric phase. In graph (c), the open squares and solid squares indicate the peaks associated with ferroelectric phase of the 50:50 copolymer and the 80:20 copolymer, respectively, as described in the text.

copolymers, whereas the period 2 and the mixture samples show a thermal expansion that is close to (but even lower than) the thermal expansion coefficient of the 80:20 sample. If we make the reasonable assumption that the interlayer interaction is proportional to the numbers of interfaces, it follows that the interactions between the 80:20 and 50:50 copolymers tend to reduce the thermal expansion coefficients of both materials. The temperature evolution of each phase is quite different and this leads to a complex temperature dependence of the lattice spacing in the heterogeneous samples. To understand the nature of these interactions better, we next consider the relative proportions of each phase as the samples are heated through the transition temperature. 
Table 2. Critical temperatures obtained from both XRD intensity and capacitance measurements as described in the text. The values of $T_{0}, T_{1}$, and $T_{1 / 2}$ were obtained from the XRD intensity data shown in figure 8 . The values of $T_{\mathrm{C}+}$ and $T_{\mathrm{C}-}$ were obtained from the capacitance peaks shown in figure 9.

\begin{tabular}{|c|c|c|c|c|c|}
\hline & \multicolumn{5}{|c|}{ Transition temperature } \\
\hline & \multicolumn{3}{|c|}{ XRD intensity } & \multicolumn{2}{|c|}{ Capacitance } \\
\hline & $T_{0}\left({ }^{\circ} \mathrm{C}\right)$ & $T_{1}\left({ }^{\circ} \mathrm{C}\right)$ & $T_{1 / 2}\left({ }^{\circ} \mathrm{C}\right)$ & $T_{C+}\left({ }^{\circ} \mathrm{C}\right)$ & $T_{C-}\left({ }^{\circ} \mathrm{C}\right)$ \\
\hline $50: 50$ & $9 \pm 1$ & $85 \pm 8$ & $43 \pm 2$ & $70 \pm 4$ & $59 \pm 3$ \\
\hline $80: 20$ & $-35 \pm 5$ & - & $138 \pm 12$ & $133 \pm 4$ & $100 \pm 8$ \\
\hline Period 20 & $30 \pm 5$ & $170 \pm 8$ & $57 \pm 6$ & $97 \pm 20$ & $78 \pm 5$ \\
\hline Period 10 & $9 \pm 4$ & $134 \pm 8$ & $60 \pm 6$ & $101 \pm 11$ & $75 \pm 6$ \\
\hline Period 2 & $-18 \pm 1$ & $128 \pm 10$ & $35 \pm 15$ & $114 \pm 5$ & $76 \pm 4$ \\
\hline Mixture & $-14 \pm 1$ & $131 \pm 1$ & $49 \pm 3$ & $105 \pm 6$ & $71 \pm 5$ \\
\hline
\end{tabular}

The relative proportions of each phase, as obtained from the integrated intensities of the x-ray peaks, are shown as a function of temperature in figure 8. For the pure 50:50 copolymer sample (see figure 8(a)), the data clearly show the gradual conversion from purely ferroelectric phase (solid circles) at low temperature to purely paraelectric phase (open circles) at high temperatures. The data from the pure 80:20 sample show a much more gradual conversion that is only half completed at the maximum measurement temperature of $125^{\circ} \mathrm{C}$. The rate of phase conversion differs among the samples. In the pure copolymer samples (see figures 8 (a) and (b)), we see that below $80{ }^{\circ} \mathrm{C}$ the conversion rate of the 50:50 copolymer is greater than that of the 80:20 copolymer, whereas above $80{ }^{\circ} \mathrm{C}$ the 80:20 copolymer has the greater conversion rate. The intensity data from the period 20 sample in figure 8(c) show independent phase conversion of the two compositions - the ferroelectric peak corresponding to the 50:50 copolymer spacing tracks the phase conversion rate of the pure 50:50 copolymer films, and similarly for the peak corresponding to the 80:20 composition. In contrast, the transition rates of the period 10, period 2, and the mixture samples in figures 6(c)-(e) are almost constant over the phase conversion range. This behavior is consistent with an interaction between the different materials, but it is not definitive evidence, because averaging the rates of phase conversion can lead to similar behavior. The endpoints of the phase conversion provide a more useful indicator of the effect of sample structure on the stability of the ferroelectric state.

With the complex phase conversion and thermal hysteresis phenomena, it is often not sufficient to identify a single transition temperature. Therefore, we define several useful critical temperatures as follows. The lower critical temperature $T_{0}$ is the temperature at which the paraelectric phase first appears as the sample is heated. The upper critical temperature $T_{1}$ is the maximum temperature of the ferroelectric phase. These critical temperatures were determined by extrapolating the XRD peak intensity data in figure $8-T_{0}$ by extrapolating the paraelectric peak intensity to zero, and $T_{1}$ by extrapolating the ferroelectric peak intensity to zero. The phase cross-over temperature $T_{1 / 2}$ is the temperature at which the integrated intensities of the ferroelectric and paraelectric peaks are equal. The value of $T_{1 / 2}$ indicates the effective midpoint of the phase coexistence region, whether or not the layers are interacting. The critical temperatures of all samples are listed in table 2. The critical temperatures of the pure 50:50 sample determined by this method are $T_{0}=9 \pm 1{ }^{\circ} \mathrm{C}$ and $T_{1}=85 \pm 8{ }^{\circ} \mathrm{C}$. These limits are clear and unambiguous because the phase coexistence region is relatively narrow and the phase transition tem- 
perature is low. However, the more gradual transition of the 80:20 sample, together with the high value of the phase transition temperature, lead to more uncertainty in the values of $T_{0}$ and $T_{1}$. Even at $125{ }^{\circ} \mathrm{C}$, the sample consists of approximately $50 \%$ ferroelectric phase and 50\% paraelectric phase, so we are not able to determine $T_{1}$ for the $80: 20$ sample. At the lowest temperature of $-5^{\circ} \mathrm{C}$, the paraelectric peak is already evident (see figure 6(b)), and $T 0$ of the 80:20 copolymer determined by this extrapolation method ranges between -40 and $-30{ }^{\circ} \mathrm{C}$.

The transition temperatures listed in table 2 indicate that, for the period 10 sample, the value of the lower critical temperature $T_{0}$ is close to that of the pure 50:50 sample, whereas for the period 2 and mixture samples, $T_{0}$ is lower, and in between that of the 50:50 and 80:20 copolymers. The value of the upper critical temperature $T_{1}$ for all three samples is approximately the same, and well above that for the pure 50:50 sample. The phase transition region in the multilayer and mixture samples is much wider than for the pure 50:50 sample, but much narrower than that of the 80:20 sample. The effect of sample structure on the transition temperature is strong evidence for interlayer interaction; the ferroelectric-to-paraelectric phase transition in these films is strongly influenced by the proximity of the $80: 20$ and 50:50 copolymers, leading to a behavior intermediate to that of the pure samples. Although we do not have enough quantitative information to extract an exact interaction length, we see that as the number of interfaces increases in going from the period 20 to period 2 sample, $T_{0}$ drops quite dramatically.

\subsection{Capacitance results}

Another indicator of the ferroelectric-paraelectric phase transition is the dielectric anomaly, a peak that occurs at or near the phase transition temperature. Figure 9 shows the dependence of the capacitance on temperature for all samples as they were heated from room temperature to at least $125^{\circ} \mathrm{C}$ and then cooled back to room temperature, in both directions at a rate of $1{ }^{\circ} \mathrm{C} \mathrm{min}^{-1}$. Decreasing the heating or cooling rates by a factor of 5 does not significantly affect the results, so we consider these measurements to be made under quasi-equilibrium conditions [20]. The capacitance data for the pure 50:50 sample (see figure 9(a)) show the typical behavior of a ferroelectric polymer, with a peak on heating indicating the ferroelectric-paraelectric phase transition, and another one on cooling indicating the paraelectric-ferroelectric phase transition. The heating and cooling curves show thermal hysteresis, a consequence of the first-order nature of the phase transition. The temperatures of the heating and cooling peaks in the capacitance, designated as $T_{\mathrm{C}+}$ and $T_{\mathrm{C}-}$, respectively, are listed in table 2 . Since the pure 80:20 sample was not fully converted to the paraelectric phase, its cooling peak (see figure 9(b)) was broadened. The cooling peaks are all very similar, with $T_{\mathrm{C}-}$ values between those for the pure samples. Note that the values of $T_{1 / 2}$ obtained from XRD are all lower than $T_{\mathrm{C}-}$. The breadth of the transition and the large thermal hysteresis are not very sensitive to sample crystallinity [51-54], as is evident by the similar shape of the capacitance-temperature curves obtained with polymorphous samples [59], LB films [47], and high-quality crystals [63].

Although the locations of the capacitance peaks in the different samples gives some indication of interaction between layers of different compositions, the shapes of the heating peaks are even more revealing. Consider the shapes of the heating peaks for the multilayer and mixture samples, as shown in figures 9(c)-(f). The period 20 multilayer sample shows a broad plateau in the capacitance on heating, whereas the period 10 sample shows a narrower plateau and the period 2 and mixture samples show rounded peaks. The expected dependence of the capacitance on temperature in an ideal non-interacting sample can be calculated by treating the layers with different compositions as capacitors con- 

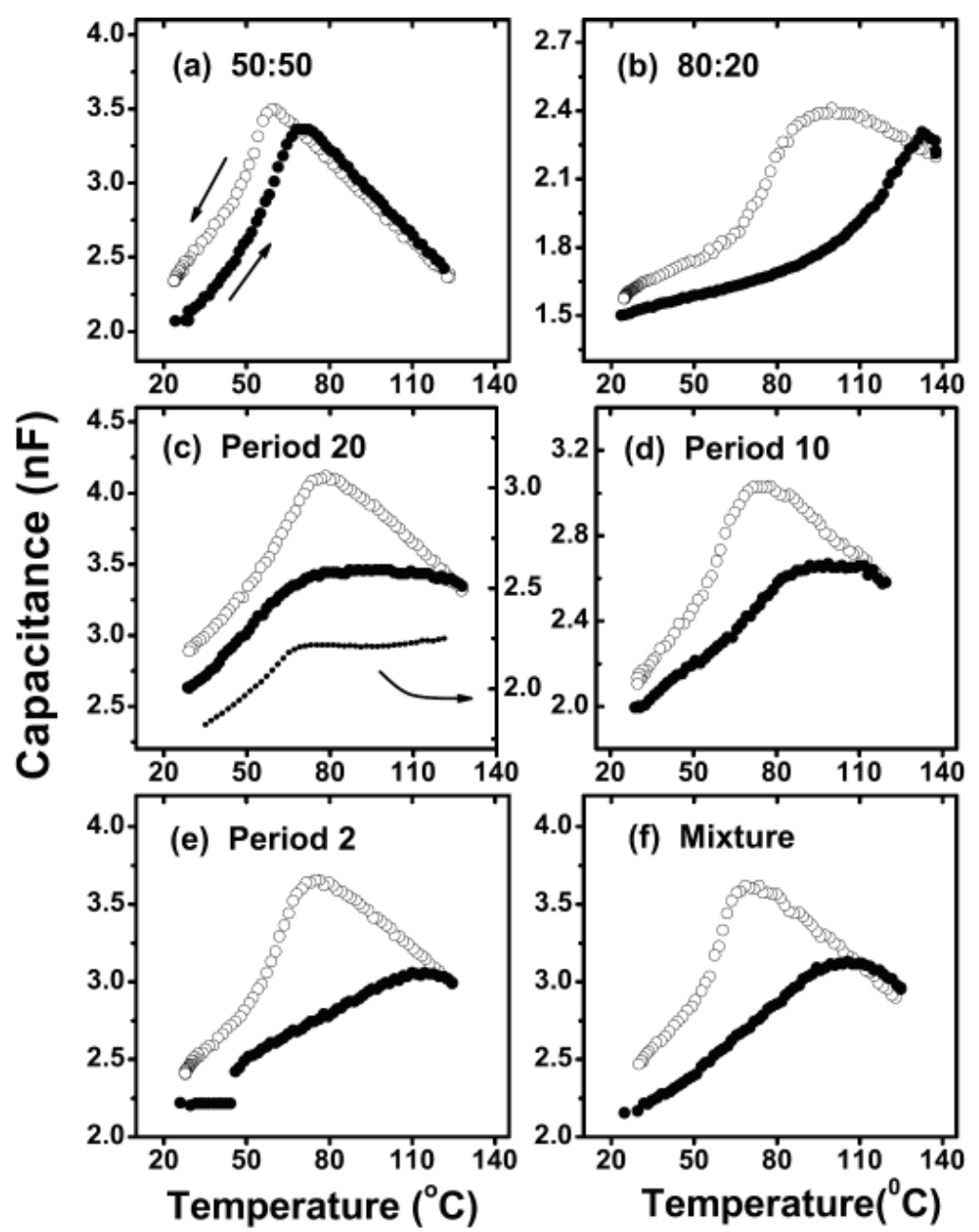

Figure 9. The dependence of capacitance on temperature during heating (solid circles) and cooling (open circles) for six capacitor samples with the following construction: (a) pure 50:50; (b) pure 80:20; (c) period 20; (d) period 10; (e) period 2; and (f) mixture.

nected in series. Applying this idealization to the data in figures 9(a) and (b) yields the dashed line shown in figure 9(c). This line would apply to any of the multilayer structures if the layers are not interacting. The calculation yields a broad plateau very similar to the one in the data for the period 20 sample, indicating that the period 20 sample behaves as two independent films with little evident interaction between them, consistent with the above analysis of the x-ray data. (The calculated capacitance is lower than that measured in the period 20 sample. Previous measurements have shown some variation in sample capacitance, probably a result of sample-to-sample variations in film thickness and electrode coverage [50].)

The period 10 sample shows a narrower plateau than expected for a non-interacting sample (i.e., the dashed line in figure $9(\mathrm{c})$ ), but not a single peak that would indicate a sample with a single transition temperature. It is more consistent with an intermediate situation - a sample consisting of independent, but interacting layers. The phase transition in part of each layer is influenced by interaction with its neighboring layers. This has the effect of raising the effective transition temperature of the adjacent 50:50 layers and lower- 
ing the effective transition temperature of the adjacent 80:20 layers and therefore narrowing the plateau compared to that of the non-interacting sample.

The heating peaks of the mixture and period 2 samples, on the other hand, are relatively narrow, consistent with a film with a single phase transition. Furthermore, the heating peaks for these samples is much closer to that of the pure 80:20 sample and there is no indication of a peak corresponding to the heating peak of the pure 50:50 sample. The capacitance and x-ray diffraction data from the mixture and period 2 samples are nearly identical to each other, further supporting the hypothesis that thin multilayers have such strong interaction that they behave as a single composite sample.

\subsection{Summary of results and discussion}

The results of the x-ray diffraction and dielectric measurements can be summarized as follows. The period 20 sample can be considered as two independent films. The temperature dependences of both the x-ray diffraction and capacitance data are consistent with two independent layers exhibiting little or no interaction. On the other end of the range, both the period 2 and mixture samples behave like a composite system with a single ferroelectricparaelectric phase diagram. The period 10 sample, on the other hand, exhibits both composite behavior and distinct features of 80:20 and 50:50 copolymers. This suggests that the effective interaction length is approximately $11 \mathrm{~nm}$, corresponding to the thickness of one of the four distinct material layers of the period 10 sample.

An alternate explanation for the composite behavior observed with the period 2 and period 10 samples is that the layers intermixed in some way, perhaps during LB deposition or during annealing. Layer mixing during deposition might arise from gaps in individual layers, but our studies show that individual layers are continuous $[64,52,57]$ and that films of only 1 or 2 LB layers usually make good capacitors [20,65, 66], indicating that gaps and pinholes are uncommon. The film transfer ratio during constant-pressure deposition is consistently near $1: 1$, which rules out significant layer mixing or thickness variation due to removal of previous layers. The x-ray diffraction measurements at the annealing temperature show that the samples were never melted, so there was no chance of mixing in the melt, when the polymer chains are mobile. Also, melted films recrystallize into needles with completely different orientation, losing their (110) orientation [52, 57]. Because of the length and stiffness of the chains, they do not readily diffuse through each other in the solid state. This process would require highly unlikely cooperative motions of dozens to hundreds of formula units in multiple chains.

To test the sample stability during annealing, and put an upper limit on interlayer diffusion, we made additional annealing studies on the period 10 and period 20 samples after all the other measurements were completed. Since the initial annealing time was $2 \mathrm{~h}$, we subjected the period 10 and period 20 samples at $135^{\circ} \mathrm{C}$ to two additional annealing cycles of $20 \mathrm{~h}$ and one of $200 \mathrm{~h}$. (This is in addition to several hours spent at elevated temperature during the synchrotron studies.) If these long annealing times were to lead to greater inter-diffusion, we would expect both greatly decreased peak intensities (due to the larger amount of defects resulting from inter-diffusion) and, in the case of the period 20 sample, a smearing of the two resolvable ferroelectric peaks (see figure 5(c)). The two 20 $\mathrm{h}$ and the $200 \mathrm{~h}$ annealing cycles did produce some decrease in the intensity of the 50:50 $\mathrm{x}$-ray diffraction peak in the period 20 sample by amounts of $10 \%$ after $40 \mathrm{~h}$, to $20 \%$ after an additional $200 \mathrm{~h}$. Similarly, the period 10 sample exhibited decreases of $15 \%$ and $34 \%$ after 40 and $200 \mathrm{~h}$, respectively. The positions of the two ferroelectric peaks from the period 20 sample were insensitive to the extra annealing, and both peaks are clearly resolvable even after a total of $240 \mathrm{~h}$ of annealing. These extended annealing studies shows that even after 100 times longer annealing there is little or no change in the period 20 sample, so it seems very unlikely that the period 10 sample suffered diffusion effects after the 
original $2 \mathrm{~h}$ annealing treatment. Taken together, all the annealing studies allow us to conclude that it is unlikely that the observed composite behavior is due to inter-diffusion of the 80:20 and 50:50 multilayers.

\section{Conclusions}

The capacitance and x-ray diffraction data from the multilayer samples establishes a progression consistent with an interaction length of approximately $11 \mathrm{~nm}$. (This is larger than the interaction length of $\leq 4 \mathrm{~nm}$ estimated for interactions between the VDF copolymer and copper-phthalocyanine particles in a nanocomposite [37].) The period 20 sample shows little or no interaction between the relatively thick films of pure 50:50 and 80:20 copolymer. The period 10 sample exhibits behavior intermediate between that of non-interacting films and a composite film. The period 2 sample behaves as a composite sample, nearly identical in structure and behavior to that of the mixture sample.

We note that the lower critical temperature, $T_{0}$, and the higher critical temperature, $T_{1}$, occur in two different regimes. At and below $T_{0}$ the samples are all fully ferroelectric. Hence all the interactions occur between ferroelectric phases of the 50:50 and 80:20 materials. Between $T_{0}$ and $T_{1}$, there exists a heterogeneous mixture of ferroelectric and paraelectric regions. In this regime, one can envisage an interaction driven by the polarization of the ferroelectric phase on the paraelectric regions, thereby increasing $T_{1}$ and/or changing the transition rate as compared to the pure samples.

In ferroelectric thin films, there are two general interaction mechanisms - mechanical and electrostatic. Both are connected with boundary conditions and are most pronounced in systems with a first-order ferroelectric phase transition. Mechanical interaction arising from stress is common in epitaxial systems, where the interaction length is the distance over which strain is relaxed, usually through accumulation of dislocations [16, 33]. For the VDF copolymers, the lattice constants perpendicular to the polymer chain are $10 \%$ smaller in the ferroelectric phase than in the paraelectric phase. For example, the compressive stress of an 80:20 layer in its ferroelectric phase, with its smaller lattice spacing, on a 50:50 layer in its paraelectric phase would tend to stabilize the ferroelectric phase of the 50:50 material, and raise its transition temperature. This effect is also observed in high-pressure studies, where the transition temperature of the VDF copolymers increases linearly with the application of hydrostatic pressure [67].

Electrostatic interactions arise from the boundary conditions on the electric displacement. In a multilayer capacitor containing both ferroelectric and paraelectric layers, surface charge on the ferroelectric layer produces an electric field in the paraelectric layer, which in turn tends to polarize the paraelectric layer, stabilizing the ferroelectric state, and raising the transition temperature of the paraelectric layer. This effect is also observed in applied-field studies, where the transition temperature of the VDF copolymers increases linearly with the application an external electric field [54].

With either mechanism, mechanical or electrostatic, the phase transition and critical temperatures for the period 10, period 2 and mixture samples can be described as follows. For the period 10 sample, the value of $T_{0}=9 \pm 4{ }^{\circ} \mathrm{C}$. For non-interacting layers, the $\mathrm{x}$-ray peaks should be additive. A simple model in which the experimental intensities of the pure 80:20 and the pure 50:50 sample as a function of temperature are added together gives an extrapolated $T_{0}$ value $2.5 \pm 3{ }^{\circ} \mathrm{C}$, which is fairly close to $T_{0}=9 \pm 4{ }^{\circ} \mathrm{C}$. This indicates that in the ferroelectric phase, there is little or no interaction in the period 10 sample. The much lower $T_{0} \mathrm{~s}$ of the period 2 and the mixture samples is a strong indication of the existence of interaction in these samples in the ferroelectric phase. The period 10, period 2 , and mixture samples have similar transition rates over the entire transition region, with the peak intensities changing at a rate of approximately $0.008 \pm 0.002\left({ }^{\circ} \mathrm{C}\right)^{-1}$. This could 
be interpreted as evidence that in this mixed paraelectric-ferroelectric phase region, the interaction has a fairly long length scale and is insensitive to the shorter period.

In summary, studies of ferroelectric-paraelectric phase transition temperature in multilayers consisting of ferroelectric VDF copolymers with different compositions, and therefore different phase transition temperatures, show clear evidence of interaction between the two copolymers, with an estimated interaction length of $11 \mathrm{~nm}$. The main evidence for this conclusion is the observation that samples with a half period thicker than $11 \mathrm{~nm}$ exhibit little or no interaction, while shorter period samples exhibit interaction, and the sample with the shortest period exhibits composite behavior nearly identical to a mixture sample. The transition from independent to interacting to composite behavior has not been reported before with ferroelectric multilayers. This begins a new approach to the study of polarization correlations in ferroelectric systems. It also offers new opportunities for materials engineering afforded by the ability to control the transition temperature and the breadth of the coexistence region. For example, ferroelectric polymers make a good acoustic match to aqueous environments and are therefore useful for ultrasonic imaging and for sonar, where the temperature of the environment is nearly constant.

\section{Acknowledgments}

The authors are grateful to Matt Poulsen for help on the dielectric measurements and suggestions on x-ray diffraction data fitting, and to Andreas Menzel for helpful suggestions. This work was supported by the Department of Energy under grant number DEFG0201EF45891 and by the Nebraska Research Initiative. The work at Argonne National Laboratory (HY) and the use of the Advanced Photon Source were supported by the Office of Basic Energy Sciences, US Department of Energy, under contract no DE-AC02-06CH11357.

\section{References}

[1] Parkin S S P 1991 Systematic variation of the strength and oscillation period of indirect magnetic exchange coupling through the 3d, 4d, and 5d transition metals Phys. Rev. Lett. 67 3598-601

[2] Borchers J A, Carey M J, Berkowitz A E, Erwin R W, and Majkrzak C F 1993 Propagation of antiferromagnetic order across paramagnetic layers in $\mathrm{CoO} / \mathrm{NiO}$ superlattices J. Appl. Phys. 73 6898-900

[3] Borchers J A, Carey M J, Erwin R W, Majkrzak C F, and Berkowitz A E 1993 Spatially modulated antiferromagnetic order in $\mathrm{CoO} / \mathrm{NiO}$ superlattices Phys. Rev. Lett. 70 1878-81

[4] Van der Zaag P J, Ijiri Y, Borchers J A, Feiner L F, Wolf R M, Gaines J M, Erwin R W, and Verheijen M A 2000 Difference between blocking and Néel temperatures in the exchange biased $\mathrm{Fe}_{3} \mathrm{O}_{4} \mathrm{CoO}$ system Phys. Rev. Lett. 84 6102-5

[5] Tilley D R 1993 Phase Transitions in Thin Films in Ferroelectric Ceramics (Basel: Birkhäuser)

[6] Tilley D R 1996 Finite-size effects on phase transitions in ferroelectrics Ferroelectric Thin Films: Synthesis and Basic Properties ed C Paz de Araujo, J F Scott and G F Taylor (Amsterdam: Gordon and Breach) pp 11-45

[7] Scott J F 1988 Properties of ceramic $\mathrm{KNO}_{3}$ thin film memories Physica B 150 160-7

[8] Scott J F 1991 Phase transitions in ferroelectric thin films Phase Transit. 30 107-10

[9] Karasawa J, Sugiura M, Wada M, Hafid M, and Fukami T 1996 Ultra-thin lead titanate films prepared by tripole magnetron sputtering Integr. Ferroelectr. 12 105-14

[10] Ishikawa K, Yoshikawa K, and Okada N 1988 Size effect on the ferroelectric phase transition in $\mathrm{PbTiO}_{3}$ ultrafine particles Phys. Rev. B 37 5852-5

[11] Schlag S and Eicke H-F 1994 Size driven phase transition in nanocrystalline $\mathrm{BaTiO}_{3}$ Solid State Commun. 91 993-7

[12] Tanaka M and Makino Y 1998 Finite size effects in submicron barium titanate particles Ferroelectr. Lett. 2413

[13] Maruyama T, Saitoh M, Sakai I, Hidaka T, Yano Y, and Noguchi T 1998 Growth and characterization of 10nm-thick c-axis oriented epitaxial $\mathrm{PbZr}_{0.25} \mathrm{Ti}_{0.75} \mathrm{O}_{3}$ thin films on (100) Si substrates Appl. Phys. Lett. 73 3524-6

[14] Yanase N, Abe K, Fukushima N, and Kawakubo T 1999 Thickness dependence of ferroelectricity in heteroepitaxial $\mathrm{BaTiO}_{3}$ thin film capacitors Japan. J. Appl. Phys. 38 5305-8 
[15] Tybell T, Ahn C H, and Triscone J-M 1999 Ferroelectricity in thin perovskite films Appl. Phys. Lett. 75 $856-8$

[16] Pertsev N A, Contreras J R, Kukhar V G, Hermanns B, Kohlstedt H, and Waser R 2003 Coercive field of ultrathin $\mathrm{Pb}\left(\mathrm{Zr}_{0.52} \mathrm{Ti}_{0.48}\right) \mathrm{O}_{3}$ epitaxial films Appl. Phys. Lett. 83 3356-8

[17] Nagarajan V, Prasertchoung S, Zhao T, Zheng H, Ouyang J, and Ramesh R 2004 Size effects in ultrathin epitaxial ferroelectric heterostructures Appl. Phys. Lett. 84 5225-7

[18] Junquera J and Ghosez P 2003 Critical thickness for ferroelectricity in perovskite ultrathin films Nature $\mathbf{4 2 2}$ $506-9$

[19] Vanderbilt D 2004 First-principles theory of polarization and electric fields in ferroelectrics Ferroelectrics $3019-14$

[20] Bune A V, Fridkin V M, Ducharme S, Blinov L M, Palto S P, Sorokin A V, Yudin S G, and Zlatkin A 1998 Two-dimensional ferroelectric films Nature 391 874-7

[21] Fong D D, Stephenson G B, Streiffer S K, Eastman J A, Auciello O, Fuoss P H, and Thompson C 2004 Ferroelectricity in ultrathin perovskite films Science 304 1650-3

[22] Nakagawara O, Shimuta T, Makino T, Arai S, Tabata H, and Kawai T 2002 Dependence of dielectric and ferroelectric behaviors on growth orientation in epitaxial ${ }_{\mathrm{BaTiO}} / \mathrm{SrTiO}_{3}$ superlattices Vacuum 66 397-401

[23] Lichtensteiger C, Triscone J-M, Junquera J, and Ghosez P 2005 Ferroelectricity and tetragonality in ultrathin $\mathrm{PbTiO}_{3}$ films Phys. Rev. Lett. 94047603

[24] Sepliarsky M, Phillpot S R, Wolf D, Stachiotti M G, and Migoni R L 2001 Long-ranged ferroelectric interactions in perovskite superlattices Phys. Rev. B 64 060101/1-4

[25] Sepliarsky M, Phillpot S R, Stachiotti M G, and Migoni R L 2002 Ferroelectric phase transitions and dynamical behavior in $\mathrm{KNbO}_{3} / \mathrm{KTaO}_{3}$ superlattices by molecular-dynamics simulation J. Appl. Phys. 91 3165-71

[26] Li S, Eastman J A, Ventroner J M, Newnham R E, and Cross L E 1997 Dielectric response in ferroelectric superlattices Phil. Mag. B 76 47-57

[27] Specht E D, Christen H-M, Norton D P, and Boatner L A 1998 X-ray diffraction measurements of the effect of layer thickness on the ferroelectric transition in epitaxial $\mathrm{KTaO}_{3} / \mathrm{KNbO}_{3}$ multilayers Phys. Rev. Lett. 80 4317-20

[28] Jiang A Q, Scott J F, Lu H, and Chen Z 2003 Phase transition and polarizations in epitaxial $\mathrm{BaTiO}_{3} / \mathrm{SrTiO}_{3}$ superlattices studied by second-harmonic generation J. Appl. Phys. 93 1180-5

[29] Ahn C H, Rabe K M, and Triscone J-M 2004 Ferroelectricity at the nanoscale: local polarization in oxide thin films and heterostructures Science 303 488-91

[30] Dawber M, Lichtensteiger C, Cantoni M, Veithen M, Ghosez P, Johnson K, Rabe K M, and Triscone J-M 2005 Unusual behavior of the ferroelectric polarization in $\mathrm{PbTiO}_{3} / \mathrm{SrTiO}_{3}$ superlattices Phys. Rev. Lett. 95 177601

[31] Christen H M, Specht E D, Silliman S S, and Harshavardhan K S 2003 Ferroelectric and antiferroelectric coupling in superlattices of paraelectric perovskites at room temperature Phys. Rev. B 68 20101-1-4

[32] Ong L-H, Osman J, and Tilley D R 2002 Dielectric hysteresis loops of first-order ferroelectric bilayers and antiferroelectrics Phys. Rev. B 65 134108/1-10

[33] Pertsev N A, Tagantsev A K, and Setter N 2000 Phase transitions and strain-induced ferroelectricity in $\mathrm{SrTiO}_{3}$ epitaxial thin films Phys. Rev. B 61 R825-9

[34] Kohlstedt H, Pertsev N A, and Waser R 2002 Size effects on polarization in epitaxial ferroelectric films and the concept of ferroelectric tunnel junctions including first results Ferroelectric Thin Films: Mater. Res. Soc. Proc. (Boston, Nov. 2001) vol 695 ed S R Gilbert et al (Warrendale, PA: Materials Research Society) pp 161-72

[35] Pertsev N A, Kukhar V G, Kohlstedt H, and Waser R 2003 Phase diagrams and physical properties of single-domain epitaxial $\mathrm{Pb}(\mathrm{Zr}, \mathrm{Ti}) \mathrm{O}_{3}$ thin films Phys. Rev. B 67 054107-10

[36] Zhang Q M, Li H, Poh M, Xia F, Cheng Z-Y, Xu H, and Huang C 2002 An all-organic composite actuator material with a high dielectric constant Nature 419 284-7

[37] Li J 2003 Exchange coupling in P(VDF-TrFE) copolymer based all-organic composites with giant electrostriction Phys. Rev. Lett. 90217601

[38] Kim L, Kim J, Kim Y S, Jung D, Park N, and Lee J 2002 Structural and dielectric properties of $\mathrm{BaTiO}_{3}$ / $\mathrm{SrTiO}_{3}$ artificial superlattice on $\mathrm{MgO}$ and $\mathrm{SrTiO}_{3}$ single crystal substrates Integr. Ferroelectr. 50 219-28

[39] $\mathrm{Lu} \mathrm{Y} 2004$ Dielectric and ferroelectric behaviors in $\mathrm{Pb}\left(\mathrm{Mg}_{1 / 3} \mathrm{Nb}_{2 / 3}\right) \mathrm{O}_{3}$ - $\mathrm{PbTiO} 3$ rhombohedral/tetragonal superlattices Appl. Phys. Lett. 85 979-81

[40] O'Neill D, Bowman R M, and Gregg J M 2000 Dielectric enhancement and Maxwell-Wagner effects in ferroelectric superlattice structures Appl. Phys. Lett. 77 1520-2

[41] Zheng H, Wang J, Lofland S E, Ma Z, Mohaddes-Ardabili L, Zhao T, Salamanca-Riba L, Shinde S R, Ogale S B, Bai F, Viehland D, Jia Y, Schlom D G, Wuttig M, Roytburd A, and Ramesh R 2004 Multiferroic Ba$\mathrm{TiO}_{3}-\mathrm{CoFe}_{2} \mathrm{O}_{4}$ nanostructures Science 303 661-3

[42] Morrison F D et al 2003 Ferroelectric nanotubes reviews on advanced materials Science 4 114-22

[43] Luo Y, Szafraniak I, Zakharov N D, Nagarajan V, Steinhart M, and Wehrspohn R B 2003 Nanoshell tubes of ferroelectric lead zirconate titanate and barium titanate Appl. Phys. Lett. 83 440-2 
[44] Luo Y, Szafraniak I, Nagarajan V, Wehrspohn R B, Steinhart M, and Wendorff J H 2003 Ferroelectric lead zirconate titanate and barium titanate nanotubes Integr. Ferroelectr. 59 1513-20

[45] Nakhmanson S M, Calzolari A, Meunier V, Bernholc J, and Nardelli M B 2003 Spontaneous polarization and piezoelectricity in boron nitride nanotubes Phys. Rev. B 67 235406-1-5

[46] Koga K, Nakano N, Hattori T, and Ohigashi H 1990 Crystallization, field-induced phase transformation, thermally induced phase transition, and piezoelectric activity in $\mathrm{P}$ (vinylidene fluoride-TrFE) copolymers with high molar content of vinylidene fluoride J. Appl. Phys. 67 965-74

[47] Ducharme S, Palto S P, and Fridkin V M 2002 Ferroelectric polymer Langmuir-Blodgett films Ferroelectric and Dielectric Thin Films ed H S Nalwa (San Diego, CA: Academic) pp 545-91

[48] Sorokin A, Palto S, Blinov L, Fridkin V, and Yudin S 1996 Ultrathin ferroelectric Langmuir-Blodgett films Mol. Mater. 6 61-7

[49] Sorokin A V, Bai M, Ducharme S, and Poulsen M 2002 Langmuir-Blodgett films of polyethylene J. Appl. Phys. 92 5977-81

[50] Bai M, Sorokin A V, Thompson D W, Poulsen M, Ducharme S, Herzinger C M, Palto S, Fridkin V M, Yudin S G, Savchenko V E, and Gribova L K 2004 Determination of the optical dispersion in ferroelectric vinylidene fluoride (70\%)/trifluoroethylene (30\%) copolymer Langmuir-Blodgett films J. Appl. Phys. 95 $3372-7$

[51] Choi J, Borca C N, Dowben P A, Bune A, Poulsen M, Pebley S, Adenwalla S, Ducharme S, Robertson L, Fridkin V M, Palto S P, Petukhova N N, and Yudin S G 2000 Phase transition in the surface structure in copolymer films of vinylidene fluoride (70\%) with trifluoroethylene (30\%) Phys. Rev. B 61 5760-70

[52] Bai M, Poulsen M, Sorokin A V, Ducharme S, and Fridkin V M 2002 Morphology, annealing, and melting of ferroelectric Langmuir-Blodgett films of vinylidene fluoride (70\%) trifluoroethylene (30\%) copolymer Conf. on Electroactive Polymers and Their Applications as Actuators, Sensors, and Artificial Muscles (Boston, Nov. 2001) (Proceedings of the Materials Research Society vol 698) ed Y Bar-Cohen et al (Warrendale, PA: Materials Research Society) pp 85-91

[53] Poulsen M 2000 Use of an external electric field to convert the paraelectric phase to the ferroelectric phase in ultra-thin copolymer films of P(VDF-TrFE) B.S. Thesis University of Nebraska-Lincoln, Lincoln, Nebraska, USA

[54] Poulsen M, Adenwalla S, Ducharme S, Fridkin V M, Palto S P, Petukhova N N, and Yudin S G 2000 Use of an external electric field to convert the paraelectric phase to the ferroelectric phase in ultra-thin copolymer films of P(VDF-TrFE) Ferroelectric Thin Films IX (Boston, Nov-Dec. 2000) (Proceedings of the Materials Research Society vol 655) ed P C McIntyre, S R Gilbert, Y Miyasaka, R W Schwartz and D Wouters

[55] Bellet-Amalric E and Legrand J F 1998 Crystalline structures and phase transition of the ferroelectric P(VDF- TrFE) copolymers, a neutron diffraction study Eur. Phys. J. B 3 225-36

[56] Thompson C, Saraf R F, and Jordan-Sweet J L 1997 Effect of surface-influenced order on thermal expansivity of polymer thin films Langmuir 13 7135-40

[57] Bai M 2002 The structure of ferroelectric PVDF/TrFE copolymer Langmuir-Blodgett films PhD Dissertation University of Nebraska-Lincoln, Lincoln

[58] Ducharme S, Bune A V, Fridkin V M, Blinov L M, Palto S P, Sorokin A V, and Yudin S 1998 Critical point in ferroelectric Langmuir-Blodgett polymer films Phys. Rev. B 57 25-8

[59] Furukawa T 1989 Ferroelectric properties of vinylidene fluoride copolymers Phase Transit. 18 143-211

[60] Legrand J F 1989 Structure and ferroelectric properties of P(VDF-TrFE) copolymers Ferroelectrics 91 303-17

[61] Tashiro K 1995 Crystal structure and phase transition of PVDF and related copolymers Ferroelectric Polymers ed H S Nalwa (New York: Dekker) pp 63-181

[62] Omote K, Ohigashi H, and Koga K 1997 Temperature dependence of elastic, dielectric, and piezoelectric properties of "single crystalline" films of vinylidene fluoride trifluoroethylene J. Appl. Phys. 81 2760-9

[63] Ohigashi H, Omote K, Abe H, and Koga K 1999 Chain motions in the paraelectric phase in single crystalline films of vinylidene fluoride and trifluoroethylene copolymer $\mathrm{P}(\mathrm{VDF} / \mathrm{TrFE})$ Japan. J. Appl. Phys. 68 $1824-7$

[64] Ducharme S, Bai M, Poulsen M, Adenwalla S, Palto S P, Blinov L M, and Fridkin V M 2001 Mesoscopic structures in two-dimensional ferroelectric polymers Ferroelectrics 252 191-9

[65] Ducharme S, Fridkin V M, Bune A V, Palto S P, Blinov L M, Petukhova N N, and Yudin S G 2000 Intrinsic ferroelectric coercive field Phys. Rev. Lett. $84175-8$

[66] Fridkin V, Iavlev A, Verkhovskaya K, Vizdrik G, Yudin S, and Ducharme S 2005 Switching in one monolayer of the ferroelectric polymer Ferroelectrics 314 37-40

[67] Bellet-Amalric E, Legrand J F, Stock-Schweyer M, and Meurer B 1994 Ferroelectric transition under hydrostatic pressure in poly(vinylidene fluoride-trifluoroethylene) copolymers Polymer 35 34-6 